NOTICE: This is the author's version of a work that was accepted for publication in Journal of Biomechanics. Changes resulting from the publishing process, such as peer review, editing, corrections, structural formatting, and other quality control mechanisms may not be reflected in this document. Changes may have been made to this work since it was submitted for publication. A definitive version was subsequently published in Journal of Biomechanics, Vol. 46, Issue 14 (2013). doi: 10.1016/j.jbiomech.2013.07.007 


\title{
Effect of the velopharynx on intraluminal pressures in reconstructed pharynges derived from individuals with and without sleep apnea
}

\author{
Julien Cisonni ${ }^{\mathrm{a}, *}$, Anthony D. Lucey ${ }^{\mathrm{a}}$, Jennifer H. Walsh ${ }^{\mathrm{b}}$, Andrew J. C. King ${ }^{\mathrm{a}}$, Novak S. J. Elliott ${ }^{\mathrm{a}}$, David D. Sampson ${ }^{\mathrm{c}}$, Peter R. \\ Eastwood $^{\mathrm{b}, \mathrm{d}}$, David R. Hillman ${ }^{\mathrm{b}}$ \\ ${ }^{a}$ Fluid Dynamics Research Group, Department of Mechanical Engineering, Curtin University, Perth, WA 6845, Australia \\ ${ }^{b}$ West Australian Sleep Disorders Research Institute, Department of Pulmonary Physiology and Sleep Medicine, Sir Charles Gairdner Hospital, Nedlands, WA \\ 6009, Australia \\ ${ }^{c}$ Optical+Biomedical Engineering Laboratory, School of Electrical, Electronic $\mathcal{G}$ Computer Engineering and Centre for Microscopy, Characterisation E Analysis, \\ The University of Western Australia, Perth, WA 6009, Australia \\ ${ }^{d}$ Centre for Sleep Science, School of Anatomy, Physiology and Human Biology, University of Western Australia, Nedlands, WA 6009, Australia
}

\begin{abstract}
The most collapsible part of the upper airway in the majority of individuals is the velopharynx which is the segment positioned behind the soft palate. As such it is an important morphological region for consideration in elucidating the pathogenesis of obstructive sleep apnea (OSA). This study compared steady flow properties during inspiration in the pharynges of 9 male subjects with OSA and 9 body-mass index (BMI)- and age-matched control male subjects without OSA. The $k$ - $\omega S S T$ turbulence model was used to simulate the flow field in subject-specific pharyngeal geometric models reconstructed from anatomical optical coherence tomography (aOCT) data. While analysis of the geometry of reconstructed pharynges revealed narrowing at velopharyngeal level in subjects with OSA, it was not possible to clearly distinguish them from subjects without OSA on the basis of pharyngeal size and shape alone. By contrast, flow simulations demonstrated that pressure fields within the narrowed airway segments were sensitive to small differences in geometry and could lead to significantly different intraluminal pressure characteristics between subjects. The ratio between velopharyngeal and total pharyngeal pressure drops emerged as a relevant flow-based criterion by which subjects with OSA could be differentiated from those without.
\end{abstract}

Keywords: Airway resistance, CFD, Obstructive sleep apnea, Pharyngeal wall pressure, Velopharynx

\section{Introduction}

Failure to maintain the patency of the upper airway during sleep characterizes obstructive sleep apnea (OSA), an extremely common and disabling disorder. This failure occurs as the result of a sleep-related loss of compensatory dilator muscle activity in individuals with anatomically predisposed airways. Many factors, including obesity and narrow skeletal confines, can contribute to this predisposition (Isono, 2012). These factors can act to both narrow the airway lumen (Rodenstein et al., 1990; Kim et al., 2008) and increase airway wall compliance (Schwab et al., 2003). The velopharyngeal airway appears to be particularly affected (Schwab et al., 1995; Arens et al., 2005). These anatomical characteristics combined with the aerodynamic forces created by inspiratory airflow through the complex airway geometry (Lucey et al., 2010) play an important role in the pathogenesis of OSA. Several studies have demonstrated the fluid-structure interaction mechanisms of upper airway collapse involved in OSA from idealized (Balint and Lucey, 2005; Chouly et al., 2008; Howell et al., 2009; Elliott et al., 2010) and realistic (Chouly et al., 2006; Zhu et al., 2012) geometric and tissue modeling.

${ }^{*}$ Corresponding author. E-mail address: julien.cisonni@gmx.com, Phone: $+61(0) 892667836$
Various imaging techniques can be used to obtain quantitative representations of an individual's airway geometry (De Backer et al., 2008). In general, previous imaging studies have shown a relationship between morphological features of the airway, such as upper airway length (Segal et al., 2008) or velopharyngeal size (Walsh et al., 2008), and the severity of OSA. However, it remains difficult to distinguish patients with OSA from healthy individuals using only geometric features of the airway (Vos et al., 2010). Further, it is generally accepted that a combination of parameters, including morphological data such as body-mass index (BMI), geometric data such as airway narrowness and flow characteristics such as airway resistance, is required to optimize OSA diagnosis and treatment evaluation (Vos et al., 2007). For example, pre- and post-treatment airway shapes, flow characteristics and apnea-hypopnea index (AHI) have been evaluated for mandibular advancement devices (MAD) (Zhao et al., 2013), nasal surgery (Wang et al., 2012) and maxillomandibular advancement (MMA) surgery (Huynh et al., 2009; Mihaescu et al., 2011). These studies have shown a relationship between the reduction of AHI and the reduction of airway resistance but they have been limited to a small number of subjects. By contrast, Van Holsbeke et al. (2011) have used statistical analyses with a large number of subjects to identify the types of patients who would most benefit from mandibular repositioning (MR) to decrease airway re- 
sistance.

Direct numerical simulations (DNS) have revealed the very different flow patterns which can appear within the airway due to the complexity of the airway shape and the inter-subject variability (Nicolaou and Zaki, 2013). However, the use of largeeddy simulation (LES) or steady Reynolds-averaged NavierStokes (RANS) turbulence models can reduce the computational cost to simulate the flow within the airway and give accurate predictions of important flow features (Mihaescu et al., 2008; Cui and Gutheil, 2011). The validity of these models has been confirmed against in-vitro measurements in reconstructed airways (Mylavarapu et al., 2009; Kim and Chung, 2009).

Anatomical observations have shown that the velopharynx tends to be narrower for patients with OSA (Walsh et al., 2008) and simulations have demonstrated that the narrowing of the velopharyngeal cross-section formed by the soft palate and posterior pharyngeal wall generates strong pressure gradients within this part of the pharynx and leads to an increase in airway resistance (Lucey et al., 2010). Flow simulations are thought to yield a stronger indicator of propensity to OSA than anatomical features because of the nonlinear relationship between geometric and flow characteristics within the pharynx (Nicolaou and Zaki, 2013). The main focus of the present study was therefore to determine the influence of velopharyngeal shape and size on the pressure drop across the pharynx and to evaluate the capacity of flow characteristics to identify individuals with and without OSA. Our hypothesis was that study of wakeful upper airway flow characteristics would more accurately distinguish such individuals than examination of airway dimensions alone.

\section{Methods and materials}

A comparison was made between the steady flow properties during inspiration in the reconstructed pharynges of nine subjects with OSA and nine control subjects without OSA.

\subsection{Subjects}

The subjects belonging to the OSA group (subjects A1 to A9) were recruited from volunteer patients who had undergone a clinic-based polysomnogram that diagnosed or confirmed OSA $($ AHI $>10)$. None had received any treatment for OSA nor undergone upper airway surgery.

The subjects belonging to the control group (subjects $\mathrm{C} 1$ to C9) were recruited from volunteers belonging to local service clubs matching the BMI and age values of the OSA group. None had a history of habitual snoring. They underwent a laboratory-based polysomnogram over a full night to confirm the absence of OSA.

The subjects of both groups were males and otherwise healthy. Subjects' age, BMI and AHI are reported in Table 1. The Human Research Ethics Committee at Sir Charles Gairdner Hospital approved the project and informed written consent was obtained from all participants.



Figure 1: Schematic representation of the human upper airway in the midsagittal plane.

\subsection{Measurements}

The airway geometry was measured with anatomical optical coherence tomography (aOCT), a minimally invasive endoscopic technique based on OCT with a near-infrared light, specifically adapted to map the anatomy of internal organs such as the airway (Armstrong et al., 2003, 2006; Leigh et al., 2008; Walsh et al., 2008). The system consisted of an optical probe placed inside a sealed, transparent catheter, with a $3 \mathrm{~mm}$ outer diameter. It operated by directing a light beam perpendicular to the catheter. The distance between the probe head and the air-tissue interface of the airway wall was determined from the reflected light using a low-coherence optical interferometer. The catheter was inserted via the nares to the level of the midesophagus ( $c f$. Fig. 1) and taped to the external nares once in position. The probe rotated at $1.25 \mathrm{~Hz}$ to capture quantitative cross-sectional images of the upper airway.

The aOCT datasets used in this study were obtained from a pullback scan made while the subject was supine, relaxed and awake. The pullback scan involved retracting the optical probe within the catheter at a constant speed of $0.2 \mathrm{~mm} . \mathrm{s}^{-1}$ from the upper esophagus to the nasal cavity. During the scan, which took approximately 12 min to complete, subjects were instructed to breathe normally via the nose, to relax their tongue with the tip resting on the posterior surface of the upper incisors to ensure that a constant tongue position was maintained during scanning, and to indicate any swallowing. Simultaneously, rib cage and abdominal motion were continuously monitored by respiratory inductance pneumography.

For each subject, the aOCT dataset included more than 1000 images representing a quantitative cross-section of the airway in the plane orthogonal to the catheter. About one third of the frames, corresponding to images acquired in the nasal cavity and in the esophagus, acquired during swallowing, or present- 
Table 1: Subject characteristics: age, body-mass index (BMI), apnea-hypopnea index (AHI), pharyngeal length $\left(L_{P}\right)$, pharyngeal average cross-sectional area $\left(\mathrm{CSA}_{\mathrm{avg}}\right)$, pharyngeal minimum cross-sectional area $\left(\mathrm{CSA}_{\min }\right)$, velopharyngeal minimum cross-sectional area $\left(\mathrm{CSA} \mathrm{AP}_{\mathrm{VP}}\right)$ and ratio between $\mathrm{CSA} \mathrm{VP}_{\mathrm{V}}$ and $\mathrm{CSA} \mathrm{A}_{\mathrm{avg}}$. Mean values and standard deviations for the control group, for the OSA group and overall are indicated, as well as the $p$-values of Student's unpaired $t$-tests comparing the OSA and control groups.

\begin{tabular}{|c|c|c|c|c|c|c|c|c|}
\hline Subject & $\begin{array}{c}\text { Age } \\
\text { [years] }\end{array}$ & $\begin{array}{c}\text { BMI } \\
{\left[\mathrm{kg} \cdot \mathrm{m}^{-2}\right]}\end{array}$ & $\begin{array}{c}\text { AHI } \\
{\left[\text { events.h }{ }^{-1}\right]}\end{array}$ & $\begin{array}{c}L_{P} \\
{[\mathrm{~mm}]}\end{array}$ & $\begin{array}{l}\mathrm{CSA}_{\mathrm{avg}} \\
{\left[\mathrm{mm}^{2}\right]}\end{array}$ & $\begin{array}{c}\mathrm{CSA}_{\min } \\
{\left[\mathrm{mm}^{2}\right]}\end{array}$ & $\begin{array}{l}\mathrm{CSA}_{\mathrm{VP}} \\
{\left[\mathrm{mm}^{2}\right]}\end{array}$ & $\begin{array}{c}\mathrm{CSA}_{\mathrm{VP}} / \mathrm{CSA}_{\mathrm{avg}} \\
{[-]}\end{array}$ \\
\hline $\mathrm{C} 1$ & 54 & 26.9 & 6.9 & 109.4 & 229.9 & 119.8 & 125.0 & 0.54 \\
\hline $\mathrm{C} 2$ & 66 & 26.7 & 0.6 & 95.1 & 193.2 & 82.7 & 119.0 & 0.62 \\
\hline C3 & 37 & 31.3 & 1.1 & 94.3 & 187.6 & 87.3 & 99.9 & 0.53 \\
\hline $\mathrm{C} 4$ & 38 & 21.1 & 0.4 & 79.0 & 249.1 & 171.9 & 171.9 & 0.69 \\
\hline C5 & 65 & 26.6 & 1.6 & 93.0 & 273.5 & 125.9 & 125.9 & 0.46 \\
\hline C6 & 70 & 25.5 & 7.7 & 105.3 & 498.3 & 278.2 & 286.2 & 0.57 \\
\hline C7 & 61 & 23.6 & 3.3 & 90.1 & 335.6 & 208.1 & 208.1 & 0.62 \\
\hline $\mathrm{C} 8$ & 69 & 25.6 & 1.6 & 92.2 & 235.1 & 68.0 & 123.0 & 0.52 \\
\hline C9 & 65 & 29.2 & 1.7 & 96.2 & 275.6 & 144.6 & 144.6 & 0.52 \\
\hline control & 58.3 & 26.3 & 2.8 & 95.0 & 275.3 & 143.0 & 156.0 & 0.56 \\
\hline group & \pm 12.7 & 2.9 & 2.7 & 8.7 & \pm 95.0 & \pm 67.6 & \pm 58.7 & \pm 0.07 \\
\hline A1 & 49 & 30.9 & 33.6 & 101.6 & 196.1 & 86.1 & 86.1 & 0.44 \\
\hline A2 & 43 & 42.8 & 107.6 & 101.8 & 228.8 & 138.5 & 138.5 & 0.61 \\
\hline A3 & 66 & 28.8 & 15.0 & 95.0 & 208.5 & 83.8 & 83.8 & 0.40 \\
\hline A4 & 76 & 26.1 & 48.4 & 99.6 & 304.5 & 194.2 & 194.2 & 0.64 \\
\hline A5 & 67 & 34.5 & 38.1 & 74.8 & 216.0 & 96.8 & 96.8 & 0.45 \\
\hline A6 & 53 & 28.7 & 40.3 & 92.8 & 324.4 & 58.0 & 58.0 & 0.18 \\
\hline A7 & 61 & 20.6 & 30.0 & 102.0 & 372.6 & 145.6 & 145.6 & 0.39 \\
\hline A8 & 49 & 23.9 & 72.1 & 91.4 & 179.5 & 64.9 & 64.9 & 0.36 \\
\hline A9 & 68 & 30.5 & 28.8 & 96.9 & 205.6 & 100.7 & 103.0 & 0.50 \\
\hline OSA & 59.1 & 29.6 & 46.0 & 95.1 & 248.5 & 107.6 & 107.9 & 0.44 \\
\hline group & \pm 11.1 & 6.4 & \pm 27.9 & 8.6 & \pm 67.7 & \pm 43.8 & \pm 43.8 & \pm 0.14 \\
\hline \multirow{2}{*}{ overall } & 58.7 & 28.0 & 24.4 & 95.0 & 261.9 & 125.3 & 131.9 & 0.50 \\
\hline & \pm 11.6 & $\pm \quad 5.1$ & \pm 29.4 & 8.4 & \pm 81.2 & \pm 58.2 & \pm 56.0 & \pm 0.12 \\
\hline$p$-value & 0.8916 & 0.1740 & 0.0003 & 0.9766 & 0.4995 & 0.2068 & 0.0664 & 0.0260 \\
\hline
\end{tabular}

ing lighting artifacts, were excluded from the analysis. Each frame was associated with a distance from the nares along the catheter and temporally aligned with the respiratory cycle (approximately 10 frames per period).

\subsection{Airway geometry reconstruction}

In order to build a 3-D model of the airway geometry during inspiration from each subject's aOCT dataset, between 10 and 20 cross-sectional images acquired at the end of inhalation (corresponding to a peak in the pneumography signal) were selected manually. These frames were chosen to obtain an accurate cross-sectional representation of important landmarks within the airway from the nasal septum to the upper esophageal sphincter.

The contour of the airway was estimated automatically from each selected frame using classical image processing techniques (noise attenuation, binarization, closing and opening, and thinning) and a spline interpolation of the extracted pixels in the local coordinates system (2-D plane perpendicular to the catheter).

During the aOCT measurements, the distance of the probe from the nares along the catheter was recorded but the exact location of the catheter within the airway was not known. In order to transform the local coordinates of the extracted airway contours in global 3-D coordinates, as shown in Fig. 2, a standardized probe path was estimated by assuming that the catheter remained rigid once taped in position at the external nares and held in place by contraction of esophageal muscle. The estimation of this generic probe path was based on other aOCT datasets for which the catheter location was obtained using a CT scan (Lucey et al., 2010), and defined with the following assumptions:

- the plane of the first extracted cross-section at the nasal end of the airway formed an angle of $75^{\circ}$ with the $x-z$ plane,

- the plane of the last extracted cross-section at the esophageal end of the airway was parallel to the $x-z$ plane,

- the angle that formed the plane of the other extracted cross-sections with the $x-z$ plane decreased quadratically as a function of the distance of the probe from the nares along the catheter, between the first and the last crosssections,

- the catheter was fixed in the $z$-direction.

Preliminary analyses showed the weak impact of the probe path estimation on the main flow quantities of interest, which varied less than $5 \%$ when the assumptions were changed within the limits of realistic geometric configurations.

The wall of the pharynx between the nasal septum and the esophageal sphincter was obtained with a cubic spline interpolation of the surface from the extracted cross-sectional contours 




Figure 2: 3-D reconstruction of the airway geometry of subject $\mathrm{C} 1$ from the estimated probe path and the contours extracted from the normal cross-sectional aOCT frames selected.

in the global coordinate system. The barycenters of the interpolated cross-sections formed the airway centerline that was used to estimate the length of the pharynx $L_{P}$ and to analyze the profiles of the flow properties along the pharynx.

\subsection{Airflow simulation}

Flow-field computations were carried out assuming a quasistatic flow in the pharynx since during quiet breathing, the airflow timescale is much shorter than that of the breathing cycle. Therefore, a static pressure-driven flow during inspiration was simulated for all subjects. These simulations were made using OpenFOAM software (Open CFD Ltd, 2011) with a finitevolume discretization of the steady RANS equations. The $k$ $\omega$ Shear-Stress Transport (SST) turbulence model was used to solve the equations with the SIMPLE algorithm. Mihaescu et al. (2008) have suggested that an unsteady LES approach should be preferred to compute the flow-field within the airway in order to obtain more accurate predictions of important flow features such as flow separation. However, the $k-\omega$ SST model was chosen in this study not only because the assumption of a quasi-steady flow was made but also because this model is appropriate to flows with curvature and adverse pressure gradients (Wilcox, 1993), and has been shown to be a good predictor of experimental measurements of wall pressure in a patient airway geometry (Mylavarapu et al., 2009).
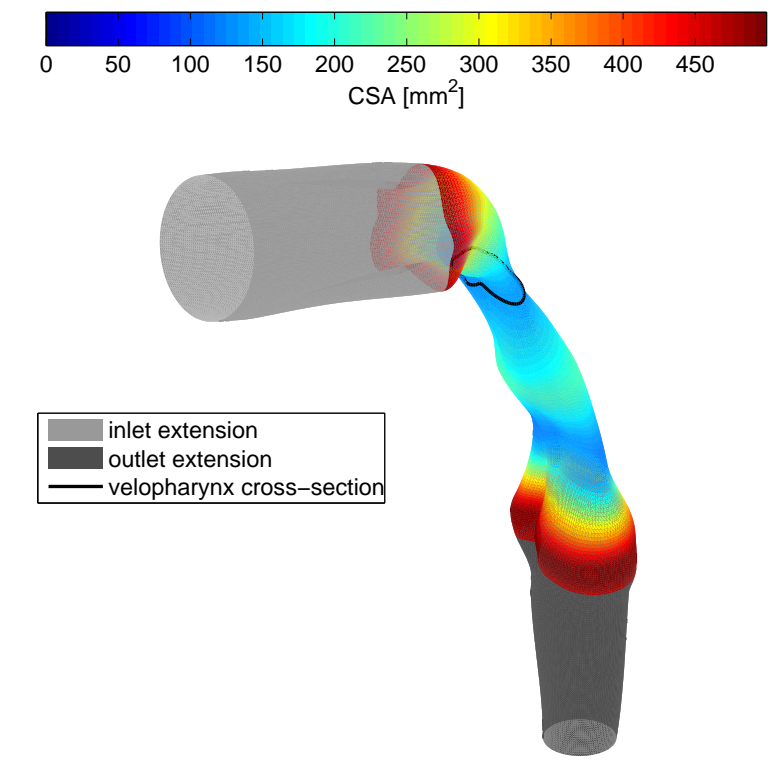

Figure 3: 3-D view of the reconstructed pharyngeal wall for subject $\mathrm{C} 1$ showing the value of the cross-sectional area (CSA) along the surface of the pharynx and the extensions at the inlet and outlet. The location of the cross-section of minimum area in the velopharynx is also indicated.

As shown in Fig. 3, extensions were added to the reconstructed pharyngeal geometries at the inlet, on the nasal end of the pharynx, and at the outlet, on the esophageal end of the pharynx. These extensions allowed the application of boundary conditions further from the reconstructed pharyngeal region to simulate more realistic flow in the region of interest in this study. The inlet extension was a tube of which the crosssectional shape was interpolated between a circle of $15 \mathrm{~mm}$ radius and the first cross-section of the reconstructed pharynx, and thus formed a converging channel. The outlet extension was a tube of which the cross-sectional shape was interpolated between the last cross-section of the reconstructed pharynx and a circle of $7.5 \mathrm{~mm}$ radius, corresponding to the typical internal radius of the trachea (Herman, 2008). The $50 \mathrm{~mm}$ length of the extensions was adapted according to the length of the reconstructed pharynges in order to obtain a constant length between the inlet and the outlet for all subjects. Thus, exactly the same boundary conditions were applied for all the simulated flowfields. A static pressure $p_{\text {inlet }}=0 \mathrm{~Pa}$ was specified at the inlet and a static pressure $p_{\text {outlet }}=-10 \mathrm{~Pa}$ was specified at the outlet (the relevance of the imposed pressure difference is discussed in Section 3.2). A no-slip boundary condition was applied at the walls.

The spatial discretization of the flow domain was made with an unstructured tetrahedral mesh. Smaller elements were defined near the walls to capture the boundary layer effects and in the regions of rapid changes in flow properties. The resulting total number of elements was between 2 and 2.5 million depending on the subject. Several preliminary simulations carried out using different mesh densities showed that the number of elements was sufficient to consider the obtained results as grid- 

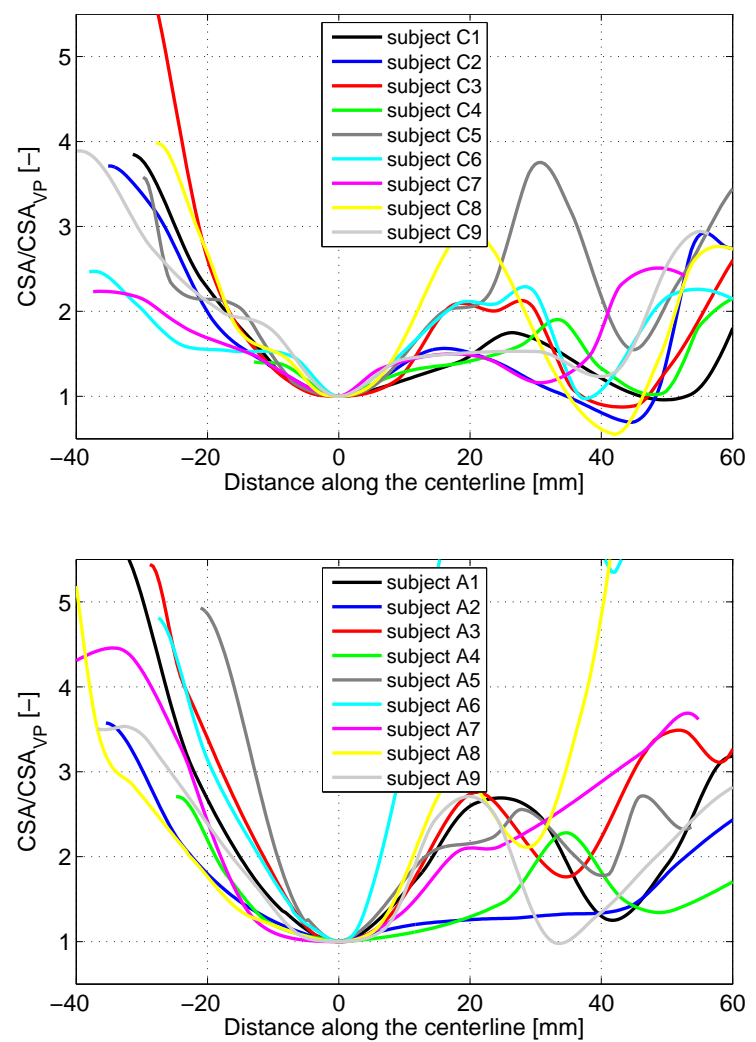

Figure 4: Comparison of the cross-sectional area profiles along the pharynx between control (top) and OSA (bottom) subjects. The values of crosssectional area (CSA) are normalized by the minimum cross-sectional area in the velopharynx $\left(\mathrm{CSA}_{\mathrm{VP}}\right)$. The distance equal to 0 corresponds to the location of the cross-section of minimum area in the velopharynx (cf. Fig. 3).

independent. This was also confirmed by the maximum values of dimensionless wall distance $\left(y^{+}<1\right.$ overall, as reported in Table 2).

\section{Results and discussion}

For all subjects, geometric and flow characteristics were only analyzed in the section corresponding to the reconstructed pharynx, as shown in Fig. 3. Characteristics in the inlet and outlet extensions were omitted. Student's unpaired $t$-tests were used to compare several geometric and flow properties between the OSA and control groups ( $p$-values are reported in Table 1 and Table 2). The significance level chosen was $p$-value $<0.05$.

\subsection{Geometric characteristics of the pharynges}

The shape of the reconstructed pharynges was smoother than that of the real morphological pharynges and the reconstructed pharyngeal geometries were therefore considered as anatomically-derived geometric models rather than anatomically-correct ones. While recognizing the estimation of the probe path and the interpolation of the cross-sectional contours and the pharyngeal wall surfaces, we contend that the reconstructed pharynges in this study were reliable approximations of each subject's real pharyngeal geometry. Since the same reconstruction process was applied to each subject dataset, the comparative approach underpinning this study allowed the effects of the approximations to be neglected in the analysis of the results.

From Table 1, it can be seen that the total length of the reconstructed pharynx $L_{P}$ was similar for all subjects. Likewise, all pharyngeal geometries were characterized by two constrictions located in the velopharyngeal and retrolingual (in the hypopharynx, approximately $40 \mathrm{~mm}$ downstream of the velopharynx) regions, as shown in Fig. 4. While the average crosssectional area $\left(\mathrm{CSA}_{\mathrm{avg}}\right)$ was only slightly lower for the OSA group than for the control group, it was clear from the crosssectional area profiles that the velopharynx formed the most severe constriction within the whole pharynx for OSA subjects $\left(\mathrm{CSA}_{\mathrm{VP}}=\mathrm{CSA}_{\min }\right.$, except for subject A9) (Arens et al., 2005; Walsh et al., 2008). By contrast, for five of the nine subjects of the control group, the most severe constriction was located in the hypopharynx $\left(\mathrm{CSA}_{\mathrm{VP}}>\mathrm{CSA}_{\min }\right.$ for subjects $\mathrm{C} 1, \mathrm{C} 2, \mathrm{C} 3$, C6 and C8).

The ratio $\mathrm{CSA}_{\mathrm{VP}} / \mathrm{CSA}_{\mathrm{avg}}$, denoting the severity of the velopharyngeal constriction, was significantly reduced in the OSA group $(0.44 \pm 0.14)$ relative to controls $(0.56 \pm 0.07)$ $(p$-value $=0.026)$. However, the difference between the two groups remained relatively small and two OSA subjects had ratios above the overall mean value of 0.50 while one control subject had a ratio below this value. It was therefore difficult to make a very clear distinction between OSA and control subjects based upon simple geometric features of the reconstructed pharynges (Vos et al., 2010).

\subsection{Flow-field characteristics}

Flow simulations of the 3-D flow-field within the reconstructed pharynx were conducted for all subjects and the main flow characteristics are summarized in Table 2. For all the cases, the flow was pressure-driven with the same constant pressure difference of $\Delta P_{\text {tot }}=-10 \mathrm{~Pa}$ across the pharynx. Therefore, the flow-rate $Q$ for each subject depended only on the geometric configuration of the pharynx. The range of obtained flow-rates was relatively wide (12.6-33.5 $1 . \mathrm{min}^{-1}$ ) but the overall average value of $22.21 \mathrm{~min}^{-1}$ corresponded to a typical flowrate during quiet breathing. Similarly, the range of resulting Reynolds numbers (1474-2462), based on mean flow velocity and hydraulic diameter at the cross-section of minimum area in the velopharynx, and defined as:

$$
\mathrm{Re}=\frac{Q}{v \mathrm{CSA}_{\mathrm{VP}}} \frac{4 \mathrm{CSA}_{\mathrm{VP}}}{\mathrm{CSP}_{\mathrm{VP}}}=\frac{4 Q}{v \mathrm{CSP}_{\mathrm{VP}}},
$$

where $\mathrm{CSP}_{\mathrm{VP}}$ denotes the perimeter of the cross-section of minimum area in the velopharynx and $v$ the kinematic viscosity of air $\left(1.52 \times 10^{-5} \mathrm{~m}^{2} \cdot \mathrm{s}^{-1}\right)$, corresponded to that observed during quiet breathing (Van Hirtum et al., 2007; Nicolaou and Zaki, 2013). The total pharyngeal resistance:

$$
R_{\mathrm{tot}}=\frac{\Delta P_{\mathrm{tot}}}{Q}=\frac{p_{\text {inlet }}-p_{\text {outlet }}}{Q}
$$


Table 2: Characteristics of the flow field simulated for each subject: flow-rate $(Q)$, Reynolds number $(\operatorname{Re}, c f$. Eq. 1$)$, maximum velocity magnitude $\left(U_{\text {max }}\right)$, maximum dimensionless wall distance $\left(y_{\max }^{+}\right)$, minimum pressure $\left(p_{\min }\right)$, average pressure on the surface of the pharynx downstream of the velopharynx $\left(P_{\mathrm{VP}-\text { avg }}\right)$, total pharyngeal resistance $\left(R_{\mathrm{tot}}, c f\right.$. Eq. 2$)$ and ratio between the average pressure drop in the velopharynx $\left(\Delta P_{\mathrm{VP}}, c f\right.$. Table 3$)$ and the total pressure drop across the pharynx $\left(\Delta P_{\text {tot }}\right)$. Mean values and standard deviations for the control group, for the OSA group and overall are indicated, as well as the $p$-values of Student's unpaired $t$-tests comparing the OSA and control groups.

\begin{tabular}{|c|c|c|c|c|c|c|c|c|}
\hline Subject & $\begin{array}{c}Q \\
{\left[1 . \mathrm{min}^{-1}\right]}\end{array}$ & $\begin{array}{l}\mathrm{Re} \\
{[-]}\end{array}$ & $\begin{array}{c}U_{\max } \\
{\left[\mathrm{m} \cdot \mathrm{s}^{-1}\right]}\end{array}$ & $\begin{array}{c}y_{\max }^{+} \\
{[-]} \\
\end{array}$ & $\begin{array}{l}p_{\min } \\
{[\mathrm{Pa}]}\end{array}$ & $\begin{array}{c}P_{\mathrm{VP}-a v g} \\
{[\mathrm{~Pa}]}\end{array}$ & $\begin{array}{c}R_{\mathrm{tot}} \\
{\left[\mathrm{cmH} 2 \text { O.s. } \mathrm{l}^{-1} \text { ] }\right.}\end{array}$ & $\begin{array}{c}\Delta P_{\mathrm{VP}} / \Delta P_{\mathrm{tot}} \\
{[-]}\end{array}$ \\
\hline $\mathrm{C} 1$ & 21.4 & 1882 & 3.9 & 0.5 & -11.9 & -7.7 & 0.29 & 0.65 \\
\hline $\mathrm{C} 2$ & 17.2 & 2121 & 4.9 & 0.8 & -17.7 & -7.2 & 0.36 & 0.48 \\
\hline $\mathrm{C} 3$ & 15.1 & 1956 & 4.8 & 1.1 & -19.0 & -7.5 & 0.41 & 0.53 \\
\hline $\mathrm{C} 4$ & 26.9 & 2049 & 4.2 & 0.6 & -13.2 & -5.7 & 0.23 & 0.50 \\
\hline C5 & 24.9 & 2188 & 4.0 & 0.6 & -12.7 & -7.7 & 0.25 & 0.86 \\
\hline C6 & 33.5 & 1739 & 3.7 & 0.3 & -10.2 & -3.3 & 0.18 & 0.24 \\
\hline C7 & 32.5 & 2238 & 3.8 & 0.4 & -10.2 & -5.2 & 0.19 & 0.50 \\
\hline C8 & 15.2 & 1871 & 4.6 & 1.0 & -17.4 & -6.1 & 0.40 & 0.35 \\
\hline C9 & 28.3 & 2424 & 4.2 & 0.5 & -12.2 & -7.7 & 0.22 & 0.78 \\
\hline control & 23.9 & 2052 & 4.2 & 0.6 & -13.8 & -6.5 & 0.28 & 0.54 \\
\hline group & \pm 7.1 & \pm 213 & \pm 0.4 & \pm 0.3 & \pm 3.3 & \pm 1.5 & \pm 0.09 & \pm 0.19 \\
\hline A1 & 18.5 & 2032 & 4.3 & 0.7 & -13.6 & -9.7 & 0.33 & 0.97 \\
\hline A 2 & 25.6 & 2013 & 4.7 & 0.7 & -18.9 & -8.3 & 0.24 & 0.95 \\
\hline A3 & 16.6 & 2091 & 4.7 & 0.8 & -16.9 & -9.1 & 0.37 & 0.83 \\
\hline A4 & 31.6 & 1920 & 3.7 & 0.3 & -11.3 & -5.5 & 0.19 & 0.56 \\
\hline A5 & 21.3 & 2462 & 4.4 & 0.8 & -14.5 & -8.5 & 0.29 & 0.98 \\
\hline A6 & 12.6 & 1474 & 4.3 & 1.0 & -14.2 & -9.4 & 0.48 & 1.01 \\
\hline A7 & 26.4 & 2133 & 4.0 & 0.5 & -11.4 & -8.3 & 0.23 & 0.85 \\
\hline A8 & 13.7 & 1743 & 4.4 & 0.8 & -12.1 & -10.0 & 0.45 & 1.05 \\
\hline A9 & 19.2 & 2013 & 4.7 & 1.0 & -20.3 & -9.3 & 0.32 & 0.86 \\
\hline OSA & 20.6 & 1987 & 4.4 & 0.7 & -14.8 & -8.7 & 0.32 & 0.90 \\
\hline group & \pm 6.2 & \pm 271 & \pm 0.3 & \pm 0.2 & \pm 3.2 & \pm 1.3 & \pm 0.10 & \pm 0.15 \\
\hline \multirow{2}{*}{ overall } & 22.2 & 2019 & 4.3 & 0.7 & -14.3 & -7.6 & 0.30 & 0.72 \\
\hline & \pm 6.7 & \pm 239 & \pm 0.4 & \pm 0.2 & $\pm \quad 3.2$ & \pm 1.8 & \pm 0.09 & \pm 0.25 \\
\hline$\overline{p p \text {-value }}$ & 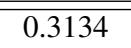 & 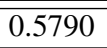 & 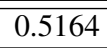 & 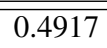 & 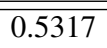 & 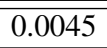 & 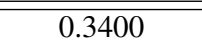 & 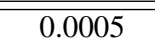 \\
\hline
\end{tabular}

gave values in the range $0.18-0.48 \mathrm{cmH} 2 \mathrm{O} . \mathrm{s} .1^{-1}$, which appeared to be low compared to most values reported in the literature (Herman, 2008; White et al., 1985; Tamisier et al., 2000). However, these values were meaningful for the measurement conditions (awake subjects breathing normally via the nose) and when considering that only a portion of the pharynges was reconstructed (Fitzpatrick et al., 2003). Moreover, the values of maximum velocity magnitude $U_{\max }$, in the range $3.7-4.9 \mathrm{~m} . \mathrm{s}^{-1}$, aligned well with those reported in other computational studies on quiet breathing with both anatomically-correct (Mihaescu et al., 2008; Nicolaou and Zaki, 2013) and idealized (Cui and Gutheil, 2011) airway geometries.

The flow characteristics $Q, \operatorname{Re}, U_{\mathrm{max}}, R_{\mathrm{tot}}$ and the minimum pressure $p_{\text {min }}$ observed in the simulated flow-fields did not appear to be significantly different for OSA and control subjects ( $p$-value $>0.3$ ). By contrast, a very obvious distinction between the two groups emerged from the pressure profiles along the pharynx, as shown in Fig. 5. This figure presents the variation of the normalized pressure:

$$
p^{\star}=\frac{p-p_{\text {outlet }}}{p_{\text {inlet }}-p_{\text {outlet }}}
$$

along the centerline of each reconstructed pharynx. The pressure profiles for the control group were characterized by a gradual pressure decrease or by two pressure drops, one in the velopharynx and one in the hypopharynx, whereas for the OSA group, the pressure profiles were characterized by only one main pressure drop in the velopharynx. The severity of the velopharyngeal constriction in subjects with OSA increased the influence of the velopharynx on the total pressure drop across the pharynx. As a consequence, the pressure downstream of the velopharynx was closer to the outlet pressure and hence much lower than that of control subjects. The effect of the severity of the velopharyngeal constriction on the pressure along the centerline applied also to the surface pressure on the pharyngeal wall, as shown in Fig. 6.

\subsection{Velopharyngeal pressure drop}

Table 3 shows the pressure fields in the velopharynx at the cross-section of minimum area (indicated in Fig. 3 and corresponding to the distance along the centerline equal to 0 in Fig. 4 and 5). For all subjects, strong cross-flow pressure gradients appeared in this region of the pharynx as has been previously demonstrated by Lucey et al. (2010). The pressure gradients were stronger for OSA subjects since the difference between the mean values of the minimum and the maximum pressures within the velopharynx was larger for the OSA group (4.3 Pa) than for the control group (2.8 Pa). For most subjects, the minimum pressure was located on the anterior side of the 
Table 3: Pressure field (in Pa) in the velopharynx at the cross-section of minimum area ( $c f$. Fig. 3 and 4 ) for all subjects. The anterior side of the velopharynx (soft palate) is on the left. Mean values and standard deviations for the control group and the OSA group are also indicated.

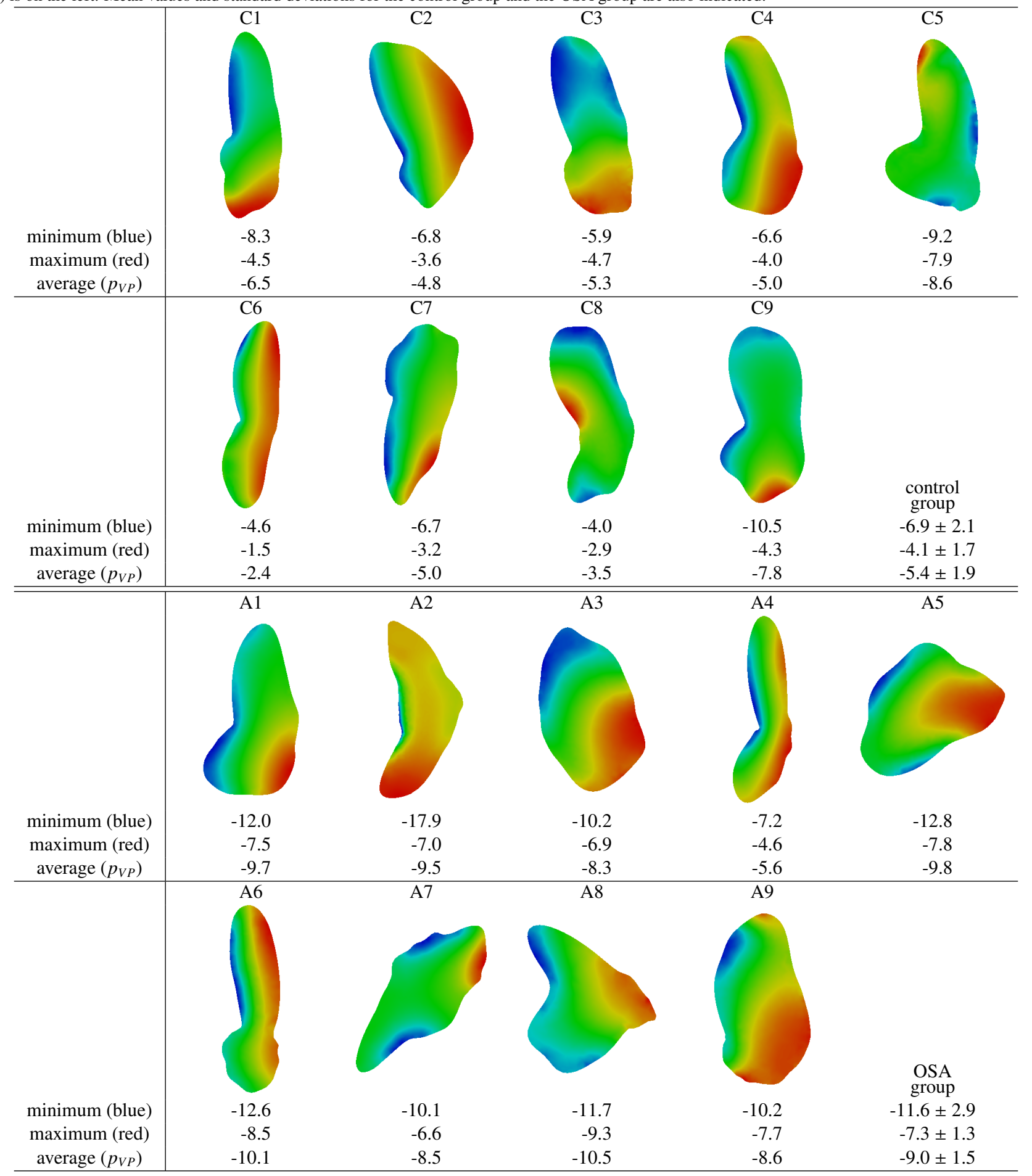

velopharynx. This contributed to a stronger suction force on the surface of the soft palate. However, the variety of shapes of the velopharyngeal constriction and of the pharynx upstream of this constriction made the cross-sectional pressure distribution very different from one individual to another. Also, due to the inertial forces in the flow, the acceleration in the velopharynx and the separation downstream of the velopharynx, the pres- sure in the velopharyngeal constriction became locally lower than $p_{\text {outlet }}=-10 \mathrm{~Pa}$ for most OSA subjects. For subjects A6 and $\mathrm{A} 8$, the cross-sectional average pressure $p_{\mathrm{VP}}$ also became lower than $p_{\text {outlet }}$. In the same way, the pressure in some parts of the pharynx downstream of the velopharynx became lower than the outlet pressure $\left(p^{\star}<0\right)$, as seen in Fig. 5.

Thus, the clearest criterion to distinguish the two groups of 

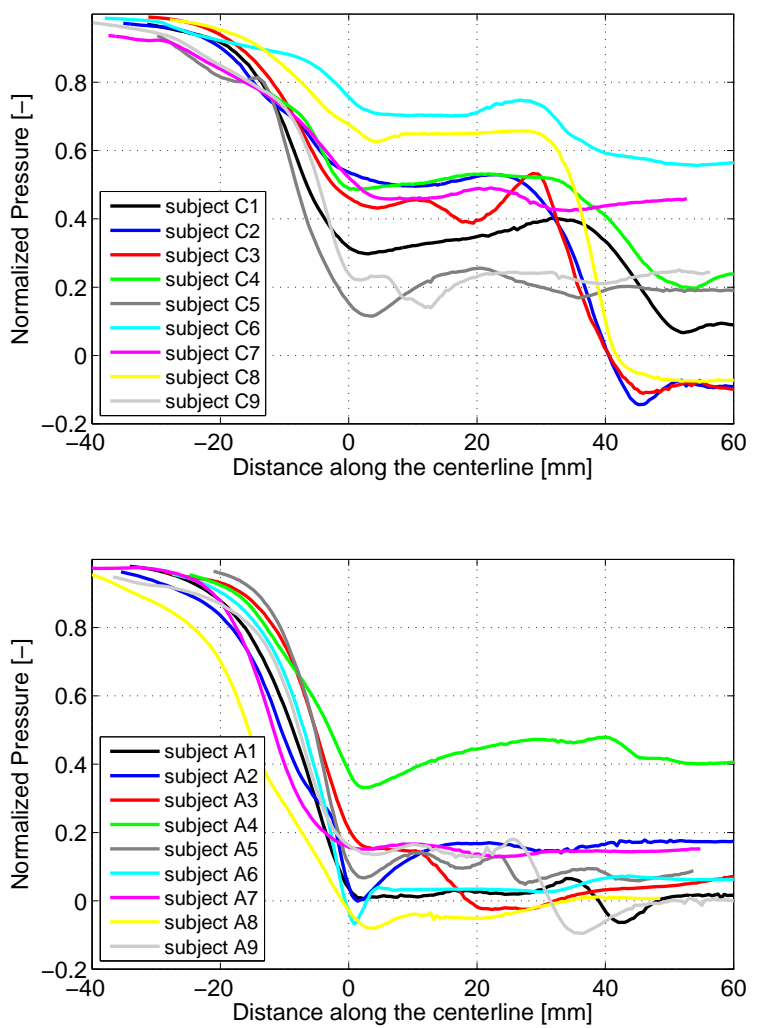

Figure 5: Comparison of the normalized pressure (cf. Eq. 3) profiles along the pharynx between control (top) and OSA (bottom) subjects. The distance equal to 0 corresponds to the location of the cross-section of minimum area in the velopharynx (cf. Fig. 3).

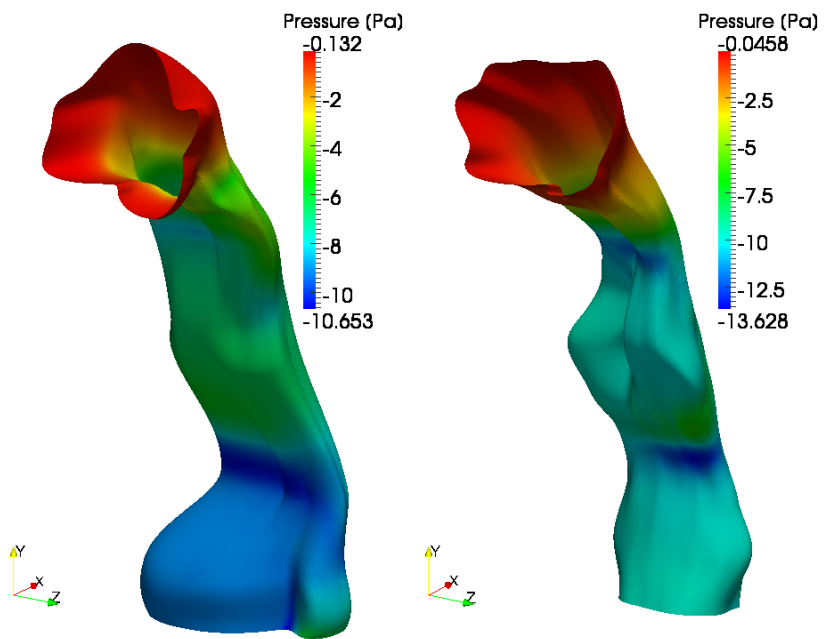

Figure 6: Pressure on the pharyngeal wall surface for subjects C1 (left) and A1 (right).

subjects remained the average pressure drop in the velopharynx $\Delta P_{\mathrm{VP}}=p_{\text {inlet }}-p_{\mathrm{VP}}(c f$. Table 2 and 3$),-5.4 \pm 1.9 \mathrm{~Pa}$ for the control group compared to $-9.0 \pm 1.5 \mathrm{~Pa}$ for the OSA group $(p$-value $=0.0005)$. The impact of the velopharynx on the total pressure drop across the pharynx could be estimated from the ratio between $\Delta P_{\mathrm{VP}}$ and $\Delta P_{\text {tot }}$. Figure 7 plots this

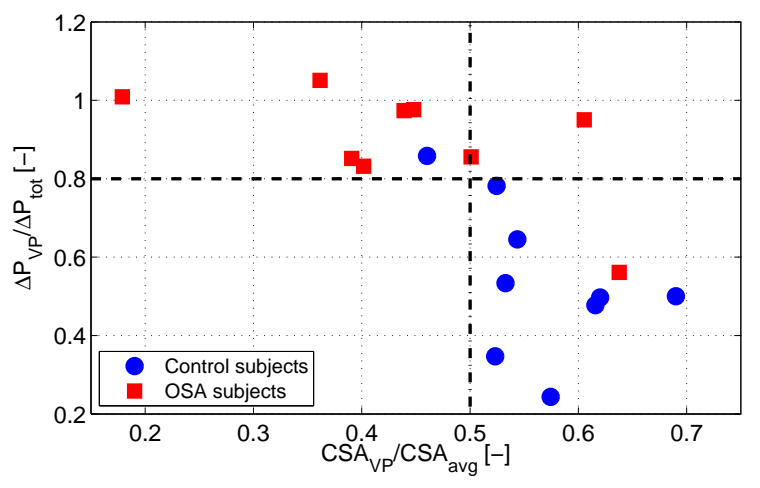

Figure 7: Ratio between the velopharyngeal pressure drop $\left(\Delta P_{\mathrm{VP}}\right)$ and the total pressure drop across the pharynx $\left(\Delta P_{\text {tot }}\right)$ as a function of the ratio between the minimum cross-sectional area in the velopharynx (CSAVP) and the average cross-sectional area of the airway $\left(\mathrm{CSA}_{\mathrm{avg}}\right)$. The values $\mathrm{CSA}_{\mathrm{VP}} / \mathrm{CSA}_{\mathrm{avg}}=0.5$ (dash-dotted line) and $\Delta P_{\mathrm{VP}} / \Delta P_{\mathrm{tot}}=0.8$ (dashed line) are also indicated.



Figure 8: Average pressure on the surface of the pharynx downstream of the velopharynx $\left(P_{\mathrm{VP}-\mathrm{avg}}\right)$ as a function of the ratio between the minimum crosssectional area in the velopharynx (CSAvP) and the average cross-sectional area of the airway $\left(\mathrm{CSA}_{\mathrm{avg}}\right)$. The inlet pressure (solid line), the outlet pressure (dashed line) and the value $P_{\mathrm{VP}-a v g}=-8 \mathrm{~Pa}$ (dash-dotted line) are also indicated.

ratio for all subjects as a function of the ratio $\mathrm{CSA}_{\mathrm{VP}} / \mathrm{CSA}_{\mathrm{avg}}$ (Van Holsbeke et al., 2011). A high degree of separation appeared between OSA and control subjects, who for the most part, fell either side of the values $\mathrm{CSA}_{\mathrm{VP}} / \mathrm{CSA}_{\mathrm{avg}}=0.5$ and $\Delta P_{\mathrm{VP}} / \Delta P_{\text {tot }}=0.8$. These qualitative thresholds strongly indicated that OSA subjects could be identified from the combination of geometric and flow parameters associated with their airway. However, further large scale statistical analyses would be necessary to refine the value of such thresholds and to provide relevant quantitative cursors for OSA detection. Nevertheless, the hypothesis that wakeful upper airway flow characteristics would more accurately distinguish OSA individuals was supported, since there was a highly significant difference between the ratios $\Delta P_{\mathrm{VP}} / \Delta P_{\text {tot }}$ for the OSA and control groups $(0.90 \pm$ 0.15 and $0.54 \pm 0.19$ respectively, $p$-value $=0.0005$ ) .

The main consequence of the high velopharyngeal pressure drop was the presence of a lower pressure field downstream of 
the velopharynx. Figure 8 plots the average pressure on the surface of the pharynx downstream of the velopharynx $P_{\mathrm{VP}-a v g}$ as a function of $\mathrm{CSA}_{\mathrm{VP}} / \mathrm{CSA}_{\mathrm{avg}}$. For the eight of the nine OSA subjects with $\Delta P_{\mathrm{VP}} / \Delta P_{\text {tot }}>0.8$, the average surface pressure was $P_{\mathrm{VP}-\text { avg }}<-8 \mathrm{~Pa}$, which corresponded to $P_{\mathrm{VP}-\text { avg }}>0.8 p_{\text {outlet }}$. Therefore, the low surface pressure obtained for the OSA group $(-8.7 \pm 1.3 \mathrm{~Pa}$ compared to $-6.5 \pm 1.5 \mathrm{~Pa}$ for the control group, $p$-value $=0.0045)$ indicated a stronger suction force exerted on the pharyngeal wall within and downstream of the velopharynx, and hence the proneness of the airway of subjects with OSA to collapse.

\section{Conclusions}

Comparison of the shape and size of the pharynx of subjects with and without OSA, reconstructed from aOCT datasets, showed that the velopharynx was the most constricted part of the pharyngeal airway for most individuals and particularly for individuals with OSA. Despite observing differences in pharyngeal geometries between a group of OSA subjects and a group of control subjects, there were not geometric measurements that allowed a clear distinction between the two groups. Steady flow simulation during inspiration using 3-D geometric models of pharynx revealed flow features that allowed subjects with OSA to be differentiated from control subjects. For the group of subjects with OSA, the pressure drop induced by the severity of the velopharyngeal constriction represented more than $80 \%$ of the total pressure drop across the pharynx and led to a lower surface pressure on the pharyngeal wall, which made the airway more prone to collapse. The ratio between velopharyngeal and total pharyngeal pressure drops emerged as a relevant flowbased criterion to differentiate subjects with OSA from control subjects.

The results obtained in this study suggest that individuals with OSA can be identified even when they are awake and breathing normally. Thus, establishing a set of simple flowbased criteria might be useful for assisting in the diagnosis and the treatment of OSA. In this regard, flow simulation using geometries reconstructed from airway tomography could be developed into a quick and convenient process to detect OSA (a complete aOCT pullback scan can take less than a minute (Jing et al., 2012) and is made while the subject is awake). Variations of flow-based parameters in an artificially deformed patient airway model could also be used to determine whether an available surgical procedure was beneficial in terms of airway flow.

\section{Acknowledgements}

The authors gratefully acknowledge financial support of the Australian Research Council through Grant DP0559408, the National Health and Medical Research Council through Grant 403953 and WA State Center of Excellence in eMedicine (Project "Airway tomography instrumentation"). The work was supported by iVEC through the use of advanced computing resources located at iVEC@Murdoch. The authors also acknowledge the assistance of Mathew Leigh in the subjectmeasurements phase of the work.

\section{Conflict of interest statement}

This manuscript has been prepared according to all ethical and scientific guidelines. No conflict of interest existed during the course of this study and the preparation of the manuscript.

\section{References}

Arens, R., Sin, S., McDonough, J. M., Palmer, J. M., Dominguez, T., Meyer, H., Wootton, D. M. and Pack, A. I.: 2005, Changes in upper airway size during tidal breathing in children with obstructive sleep apnea syndrome, Am J Resp Crit Care 171, 1298-1304.

Armstrong, J. J., Leigh, M. S., Sampson, D. D., Walsh, J. H., Hillman, D. R. and Eastwood, P. R.: 2006, Quantitative upper airway imaging with anatomic optical coherence tomography, Am J Resp Crit Care 173, 226-233.

Armstrong, J. J., Leigh, M. S., Walton, I. D., Zvyagin, A. V., Alexandrov, S. A., Schwer, S., Sampson, D. D., Hillman, D. R. and Eastwood, P. R.: 2003, In vivo size and shape measurement of the human upper airway using endoscopic long-range optical coherence tomography, Opt Express 11, 1817 1826.

Balint, T. S. and Lucey, A. D.: 2005, Instability of a cantilevered flexible plate in viscous channel flow, J Fluid Struct 20, 893-912.

Chouly, F., Van Hirtum, A., Lagre, P. Y., Paoli, J. R., Pelorson, X. and Payan, Y.: 2006, Simulation of the retroglossal fluid-structure interaction during obstructive sleep apnea, Lect Notes Comput Sc 4072, 48-57.

Chouly, F., Van Hirtum, A., Lagre, P. Y., Pelorson, X. and Payan, Y.: 2008, Numerical and experimental study of expiratory flow in the case of major upper airway obstructions with fluid-structure interaction, J Fluid Struct 24, 250269.

Cui, X. G. and Gutheil, E.: 2011, Large eddy simulation of the unsteady flow-field in an idealized human mouth-throat configuration, $J$ Biomech 44(16), 2768-2774.

De Backer, J. W., Vos, W. G., Verhulst, S. L. and De Backer, W.: 2008, Novel imaging techniques using computer methods for the evaluation of the upper airway in patients with sleep-disordered breathing: A comprehensive review, Sleep Med Rev 12, 437-447.

Elliott, N. S. J., Lucey, A. D. and Heil, M.: 2010, Large-amplitude oscillations of a finite-thickness cantilevered flexible plate in viscous channel flow, Proceedings of ASME 2010 3rd Joint US-European Fluids Engineering Summer Meeting, Montreal, Quebec, Canada.

Fitzpatrick, M. F., McLean, H., Urton, A. M., Tan, A., O’Donnell, D. and Driver, H. S.: 2003, Effect of nasal or oral breathing route on upper airway resistance during sleep, Eur Respir J 22, 827-832.

Herman, I. P.: 2008, Physics of the human body, Springer, chapter Lungs and Breathing, pp. 525-551.

Howell, R. M., Lucey, A. D., Carpenter, P. W. and Pitman, M. W.: 2009, Interaction between a cantilevered-free flexible plate and ideal flow, $J$ Fluid Struct 25, 544-566.

Huynh, J., Kim, K. B. and McQuilling, M.: 2009, Pharyngeal airflow analysis in obstructive sleep apnea patients pre- and post-maxillomandibular advancement surgery, J Fluid Eng 131.

Isono, S.: 2012, Obesity and obstructive sleep apnoea: mechanisms for increased collapsibility of the passive pharyngeal airway, Respirology 17, 3242.

Jing, J., Zhang, J., Chin L. A., Wong, B. J. F. and Chen, Z.: 2012, Highspeed upper-airway imaging using full-range optical coherence tomography, J Biomed Opt 17.

Kim, H. Y., Bok, K. H., Dhong, H.-J. and Chung, S.-K.: 2008, The correlation between pharyngeal narrowing and the severity of sleep-disordered breathing, Otolaryng Head Neck 138, 289-293.

Kim, S. and Chung, S.: 2009, Investigation on the respiratory airflow in human airway by PIV, J Visual 12(3), 259-266.

Leigh, M. S., Armstrong, J. J., Paduch, A., Walsh, J. H., Hillman, D. R., Eastwood, P. R. and Sampson, D. D.: 2008, Anatomical optical coherence tomography for long-term portable quantitative endoscopy, IEEE Trans Biomed Eng 55(4), 1438-1446.

Lucey, A. D., King, A. J. C., Tetlow, G. A., Wang, J., Armstrong, J. J., Leigh, M. S., Paduch, A., Walsh, J. H., Sampson, D. D., Eastwood, P. R. and Hillman, D. R.: 2010, Measurement, reconstruction, and flow-field computation 
of the human pharynx with application to sleep apnea, IEEE Trans Biomed Eng 57(10), 2535-2548.

Mihaescu, M., Murugappan, S., Kalra, M., Khosla, S. and Gutmark, E.: 2008, Large Eddy Simulation and Reynolds-Averaged Navier-Stokes modeling of flow in a realistic pharyngeal airway model: an investigation of obstructive sleep apnea, J Biomech 41(10), 2279-2288.

Mihaescu, M., Mylavarapu, G., Gutmark, E. J. and Powell, N. B.: 2011, Large Eddy Simulation of the pharyngeal airflow associated with obstructive sleep apnea syndrome at pre and post-surgical treatment, J Biomech 44(12), 22212228

Mylavarapu, G., Murugappan, S., Mihaescu, M., Kalra, M., Khosla, S. and Gutmark, E.: 2009, Validation of computational fluid dynamics methodology used for human upper airway flow simulations, J Biomech 42(10), 15531559.

Nicolaou, L. and Zaki, T. A.: 2013, Direct numerical simulations of flow in realistic mouth-throat geometries, J Aerosol Sci 57, 71-87.

Open CFD Ltd: 2011, OpenrFOAM, The Open Source CFD Toolbox: User Guide (Version 2.1.0), Technical report.

Rodenstein, D. O., Dooms, G., Thomas, Y., Liistro, G., Stanescu, D. C., Culee, C. and Aubert-Tulkens, G.: 1990, Pharyngeal shape and dimensions in healthy subjects, snorers, and patients with obstructive sleep apnoea, Thorax 45, 722-727.

Schwab, R. J., Gupta, K. B., Gefter, W. B., Metzger, L. J., Hoffman, E. A. and Pack, A. I.: 1995, Upper airway and soft tissue anatomy in normal subjects and patients with sleep-disordered breathing. Significance of the lateral pharyngeal walls, Am J Resp Crit Care 152, 1673-1689.

Schwab, R. J., Pasirstein, M., Pierson, R., Mackley, A., Hachadoorian, R., Arens, R., Maislin, G. and Pack, A. I.: 2003, Identification of upper airway anatomic risk factors for obstructive sleep apnea with volumetric magnetic resonance imaging, Am J Resp Crit Care 168, 522-530.

Segal, Y., Malhotra, A. and Pillar, G.: 2008, Upper airway length may be associated with the severity of obstructive sleep apnea syndrome, Sleep Breath 12, 311-316.

Tamisier, R., Pepin, J. L., Wuyam, B., Smith, R., J., A. and Levy, P.: 2000, Characterization of pharyngeal resistance during sleep in a spectrum of sleep-disordered breathing, J Appl Physiol 89, 120-130.

Van Hirtum, A., Chouly, F., Lagre, P. Y., Paoli, J. R., Payan, Y. and Pelorson, X.: 2007, Progress in Sleep Apnea Research, Nova Science Publishers, New York, chapter When a fluid-structure interaction keeps you awake: a physical approach to Obstructive Sleep Apnea, pp. 41-76.

Van Holsbeke, C., De Backer, J., Vos, W., Verdonck, P., Van Ransbeeck, P., Claessens, T., Braem, M., Vanderveken, O. and De Backer, W.: 2011, Anatomical and functional changes in the upper airways of sleep apnea patients due to mandibular repositioning: A large scale study, $J$ Biomech 44(3), 442-449.

Vos, W., De Backer, J., Devolder, A ;and Vanderveken, O., Verhulst, S., Salgado, R., Germonpre, P., Partoens, B., Wuyts, F., Parizel, P. and De Backer, W.: 2007, Correlation between severity of sleep apnea and upper airway morphology based on advanced anatomical and functional imaging, $J$ Biomech 40(10), 2207-2213.

Vos, W. G., De Backer, W. A. and Verhulst, S. L.: 2010, Correlation between the severity of sleep apnea and upper airway morphology in pediatric and adult patients, Curr Opin Allergy Clin Immunol 10(1), 26-33.

Walsh, J. H., Leigh, M. S., Paduch, A., Maddison, K. J., Philippe, D. L., Armstrong, J. J., Sampson, D. D., Hillman, D. R. and Eastwood, P. R.: 2008 Evaluation of pharyngeal shape and size using anatomical optical coherence tomography in individuals with and without obstructive sleep apnoea, J Sleep Res 17, 230-238.

Wang, Y., Wang, J., Liu, Y., Yu, S., Sun, X., Li, S., Shen, S. and Zhao, W.: 2012, Fluid-structure interaction modeling of upper airways before and after nasal surgery for obstructive sleep apnea, Int J Numer Meth Biomed Engng .

White, D. P., Lombard, R. M., J., C. R. and Zwillich, C. W.: 1985, Pharyngea resistance in normal humans: influence of gender, age, and obesity, J Appl Physiol 58, 365-371.

Wilcox, D.: 1993, Comparison of two-equation turbulence models for boundary layers with pressure gradient, AIAA $J$ 31, 1414-1421.

Zhao, M., Barber, T., Cistulli, P., Sutherland, K. and Rosengarten, G.: 2013 , Computational fluid dynamics for the assessment of upper airway response to oral appliance treatment in obstructive sleep apnea, J Biomech 46(1), 142 150.

Zhu, J. H., Lee, H. P., Lim, K. M., Lee, S. J., Teo, L. S. L. and Wang, D. Y.:
2012, Passive movement of human soft palate during respiration: A simulation of 3D fluid/structure interaction, J Biomech 45(11), 1992-2000. 